\title{
Functional and radiological ou tcome of inter-trochanteric fractures treated with minimally invasive D.H.S or proximal femoral nailing: A comparative study
}

\author{
N. Selvam ${ }^{1, *}$, K. Karthick Anand ${ }^{2}$, Amutha Ganesh ${ }^{3}$ \\ ${ }^{1}$ Associate Professor, ${ }^{2}$ Assistant Professor, ${ }^{3}$ Senior Resident, Dept. of Orthop aedic Surgery, Sri Venkataeswaraa Medical College \\ and Research Centre, Ariy ur, Pondicherry, India
}

*Corresponding Author:

Email: drnselvam@rediffmail.com

\begin{abstract}
Introduction: Inter-trochanteric fractures are one of the commonest fractures encountered in orthopaedic practice. Treatment of inter-trochanteric fractures had turned from totally conservative in the pre ' 70 s to totally surgical now. Many surgical methods like Jewet nail, Gamma nail and condy lar plates had been tried and given up. The commonest method used now are Dynamic hip screw (D.H.S) system and Proximal Femoral inter-locking nail (P.F.N) system.

Materials and Methods: This, prospective study was conducted in Sri Venkateswaraa Medical College, Pondicherry from May 2015 to May 2017. 30 (Thirty) consecutive patients of age over 55 years, with inter-trochanteric fractures were studied. Of these 30 patients alternate patients ( 15 patients) were treated with minimally invasive D.H.S and the other 15 patients were treated with P.F.N. They were reviewed every 2 weeks. Their functional results were analy sed using modified Harris hip score. ${ }^{37}$

Results: Males predominated in our study. $70 \%$ were left sided. Mean time of surgery with incision for D.H.S group was little longer than P.F.N group. P.F.N group bone weight earlier than D.H.S group. Blood loss was more in D.H.S group. There was no difference in mean hospital stay in both groups. P.F.N group patients bore weight earlier in both unstable and stable fracture groups. P.F.N group healed earlier than D.H.S group. The D.H.S group bore weight much later. There was only one complication in both groups. Bony union occurred earlier in P.F.N group than D.H.S group.

Conclusion: The P.F.N group required shorter incision and had less blood loss. They were able to bear weight earlier in both stable and unstable groups, whereas D.H.S group with unstable fractures took long time to bear weight. The P.F.N group fractures healed earlier than D.H.S group. At the end of six months, the modified Harris hip score showed no marked difference between two groups. Considering all this, we conclude PFN is a better device to treat inter trochanteric fractures especially in unstable fractures.
\end{abstract}

Keywords: Trochanteric fracture, Minimally invasive dynamic hip screw, Proximal femoral nail, Modified Harris hip score.

\section{Introduction}

Nearly $50 \%$ of fractures around hip are intertrochanteric fractures. It was a fracture of the elderly. Now-a-days because of RTA it is occurring equally in younger patients.

Till the 1980's these fractures were treated with continuous skeletal traction of 6 weeks followed by hip Spica Cast. ${ }^{18}$ This was because non-union of fracture trochanter was unheard of. But being bed ridden for months, had its own morbidity and mortality. Nearly $30 \%$ of patients succumbing to these. ${ }^{25}$

To avoid this and get the patients out of the bed early, surgical intervention with various implants were tried. Jewett and Holt ${ }^{19}$ tried with nail and plate combination. The A. $\mathrm{O}^{34}$ came later with fixed angle condylar blade plate. Results were not optimal because of fixed angle of the blade plate.

Gamma Nail ${ }^{2,422,24}$ was introduced in the 80 's. This did not last long, as the screw of the nail cut through the head of femur. ${ }^{25}$

Then came the Richards sliding hip screw ${ }^{1,6,10,34}$ (or) Dynamic hip screw system which allowed the compression and controlled collapse of the fracture. The results were encouraging.

After this, the much longer and more straighter in the proximal end of nail than the Gamma nail, called Proximal Femoral Nail ${ }^{3,8,35}$ with an additional de- rotational screw proximally was developed. It had theoretical advantage of more load transfer, with short lever arms.

Early mobilisation and quick return of function is the goal of any fracture treatment. ${ }^{9}$

With this in mind, this study was conducted to study the radiological and functional outcome of both stable and unstable inter-trochanteric fractures treated with either minimally invasive D.H.S or P.F.N and study the results.

\section{Materials and Methods}

This was a prospective study carried out at Sri Venkateswaraa Medical College and Research Centre, Ariyur, Pondicherry from May 2015 to May 2017. This study consists of 30 (Thirty) adult patients with intertrochanteric fracture. Of these 30 patients, 15 patients were treated with minimally invasive D.H.S, and 15 patients with P.F.N. Alternate patients were allotted to the respective groups irrespective of grading of the fracture. They were graded using Boyd and Griffin's classification. ${ }^{17}$ Only skeletally mature patients above the age of 18 years were included in this study. For all these patients personal data, mode of injury, type of fracture, intra and post-operative complication, duration of surgery, amount of blood loss, in the follow up when 
the patient started weight bearing, function of hip using modified Harris hip score at varying period of follow up along radiological examination were collected and analysed.

Patients with poly-trauma and multiple fractures of the same or opposite limb were excluded from the study. Patients with head injury were also excluded.

\section{Results}

Both D.H.S and P.F.N groups had 15 patients each, totalling 30 patients. Of these 30 patients, 16 were females and 14 males.

Left hip was involved in 19 cases of which 11 were treated with D.H.S and 8 with P.F.N.

Right hip was involved in 11 cases. Of which 4 were treated with D.H.S and 7 with P.F.N.

The D.H.S group had 9 patients with stable fractures and 6 with unstable fractures.

The P.F.N group had 7 patients with stable fracture and 8 with unstable fractures.

The meantime (delay time) from the time of injury to surgery was 7.2 days (Range 2 to 11 days) for minimally invasive D.H.S group and 5.8 days (Range 2 to 8 days) for the P.F.N group.

The minimally invasive surgical time was bit longer than the P.F.N group. The meantime of surgery for D.H.S group was 69.9 min (Range 60.8 to 82.5 mins) and for P.F.N group it was 52.1 min (Range 44.3 to $55.4 \mathrm{mins}$ ).

The mean blood loss was in D.H.S cases was 163 $\mathrm{ml}$ (range $85 \mathrm{ml}$ to $355 \mathrm{ml}$ ). The mean blood los s in P.F.N. cases was $97.5 \mathrm{ml}$ (range $60 \mathrm{ml}$ to $165 \mathrm{ml}$ ).

The mean length of the minimally invasive D.H.S. cases was little longer $(9.1 \mathrm{cms})$ than the P.F.N. cases (5.6 cms).

The mean blood loss and length of incision favoured almost equally with most of the comparative studies done.

Regarding per operative problems encountered, we could not get adequate and satisfactory reduction - ' $\mathrm{C}$ "arm wise in one case of D.H.S and one case of P.F.N. group. Both these cases belonged to the unstable fractures.

In the P.F.N. group - in one case we did not use the de-rotation screw for fear of breaking the superior
As D.H.S and P.F.N are common surgeries the details of them are not mentioned here.

All the patients went through the same post of rehabilitation programme till discharge.

They were requested to come at $0,4,6,8,12,16$, 20, 24 weeks interval and functional and radiological as sessment were done.

cortex of the neck and in another case the was breakage of dill bit whist locking the distal locking hole.

The mean TIP-APEX DISTANCE (TAD) ${ }^{16}$ was 16 $\mathrm{mm}$ in the D.H.S. group and for the P.F.N. group it was 17.2. mm. In the P.F.N. group TAD of Compression screw only was considered.

There was not much of the difference in the hospital stay between the two groups: 6.8 days for D.H.S group and 6.2 days for P.F.N group.

The mean time to start partial weight bearing was 3.6 weeks for both unstable and stable fractures in P.F.N group where as in D.H.S group, we allowed partial weight bearing in a mean time of 7.3 weeks for stable fractures and for unstable fractures in 12.5 weeks.

In the D.H.S group, the stable fracture healed fully in a meantime of 18.6 weeks (Range 16 weeks to 21 weeks). The unstable fractures of D.H.S group took longer time to heal fully with a mean time of 22.5 weeks (Range 20 weeks to 29 weeks).

But in the P.F.N group the union time did not differ much in both stable and unstable fracture sub-groups with 14.6 and 15.0 weeks respectively.

Eventually all the fractures in both groups healed well and none of the patients required bone grafting or any re-surgeries.

Post-operatively there was one case of infection in D.H.S which resolved with toileting and exposure to sensitive antibiotics. In the P.F.N case, there was one case of de-rotation screw cutting out.

The Mean modified Harris hip score was 69.34 for D.H.S and 81.23 for P.F $>\mathrm{N}$ group at the end of 6 weeks. But at the end of 20 weeks, there was not much of difference between D.H.S group 80.2 points and P.F.N group 81.5 points.

Our cases fared slightly better ${ }^{20}$

Table 1: Six months end hips score

\begin{tabular}{|l|c|c|}
\hline \multicolumn{1}{|c|}{ Operative Details } & Dynamic Hip Screw & Proximal Femoral Nail \\
\hline Mean time of operation after fracture in days & 7.2 & 5.8 \\
\hline Mean duration of Operation in minutes & 69.9 & 52.1 \\
\hline Stable fracture & 60.8 & 44.3 \\
\hline Unstable fracture & 82.5 & 55.4 \\
\hline Mean blood los in ml & 163 & 97.5 \\
\hline Stable fracture & 152.3 & 101.6 \\
\hline Unstable fracture & 180 & 95.7 \\
\hline Mean length of incision in cm & 9.1 & 5.6 \\
\hline Stable & 9 & 5.57 \\
\hline Unstable & 9.25 & 5.61 \\
\hline
\end{tabular}


Analysis of modified Harris hip score showed $86.6 \%$ of D.H.S group had good and excellent results. In the P.F.N group all the patients $100 \%$ had good and excellent results.

\section{DHS Case 1}

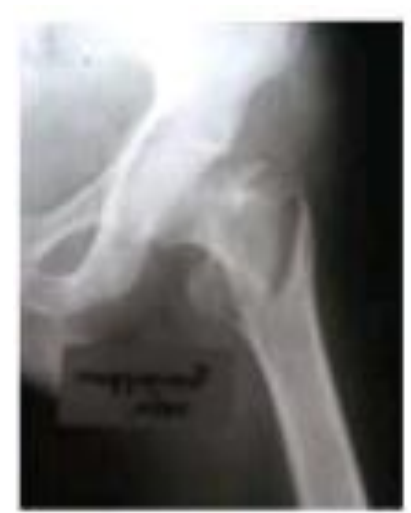

Preoperative

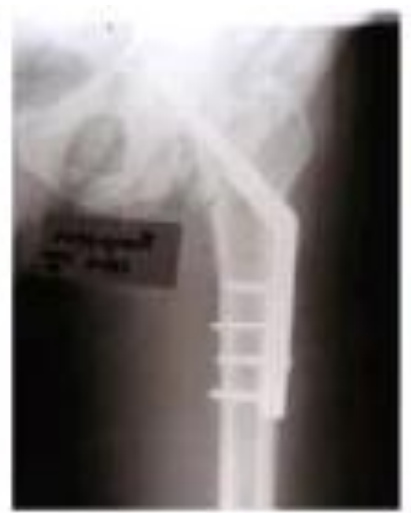

Immediate post operative

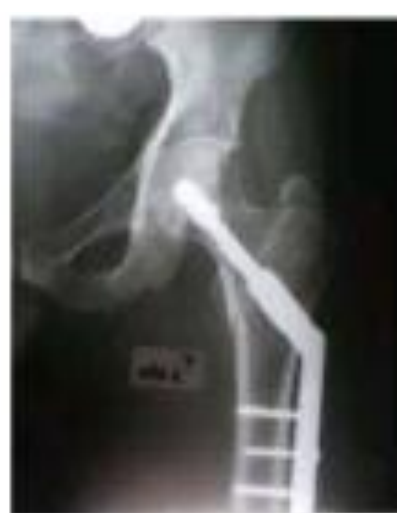

2 weeks post operative
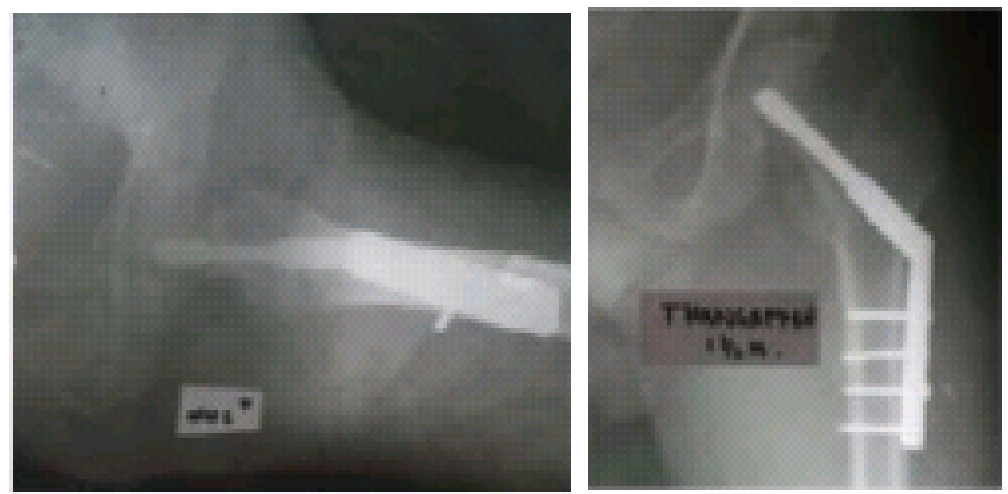

2 weeks postoperative

$1 \frac{1 / 2}{2}$ month post operative
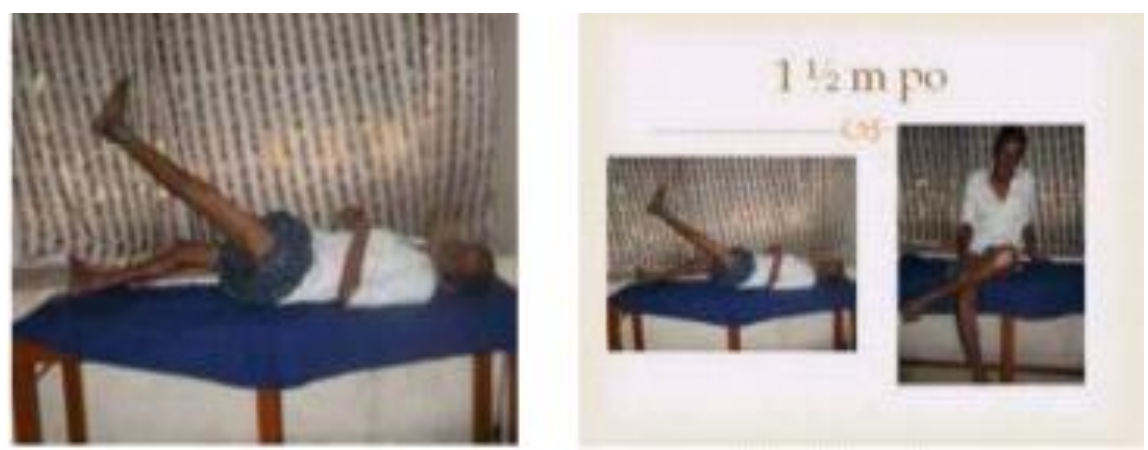

Functional outcome

Fig. 1: 
PFN Case 1:

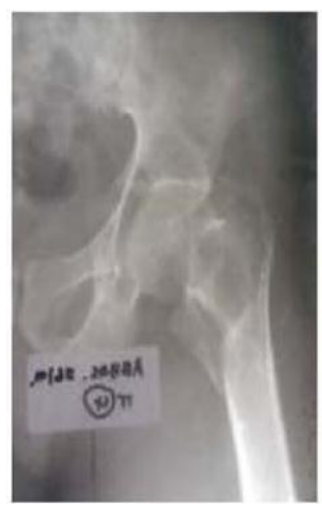

Preoperative

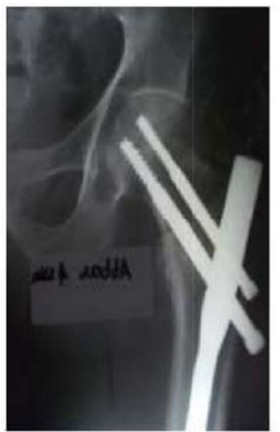

6 weeks post op

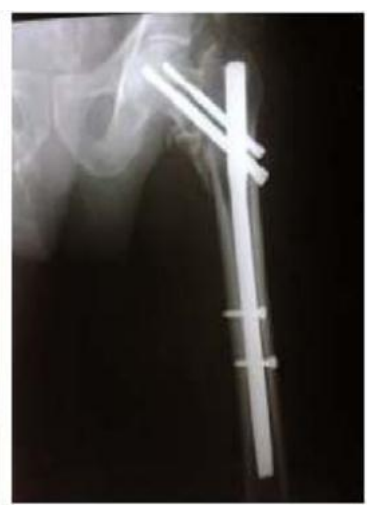

Immediate post operative
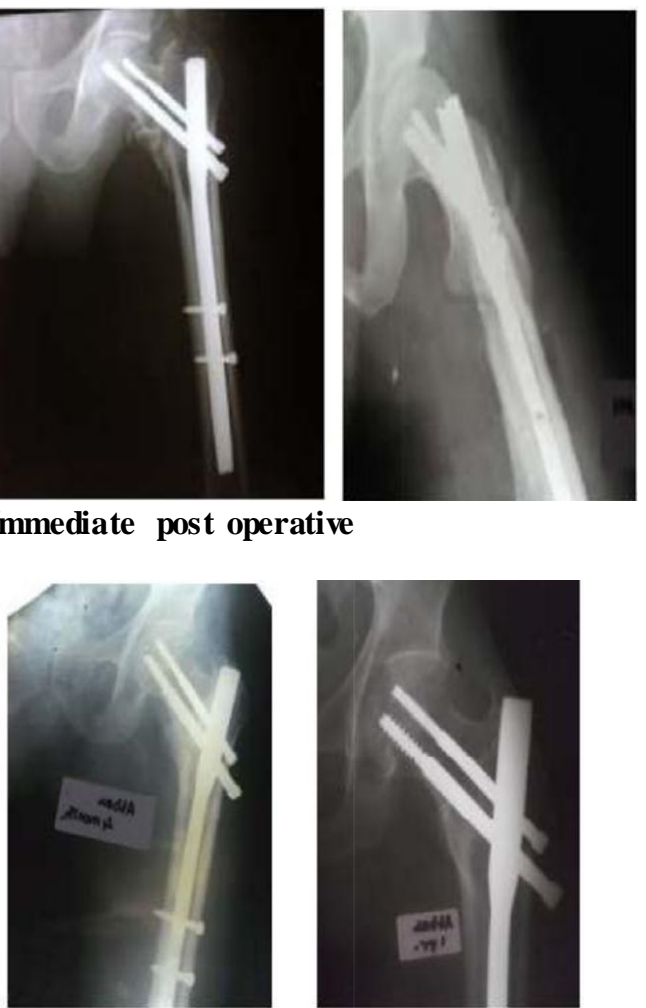

4 month post op

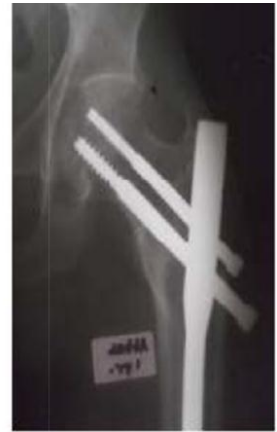

1year post op
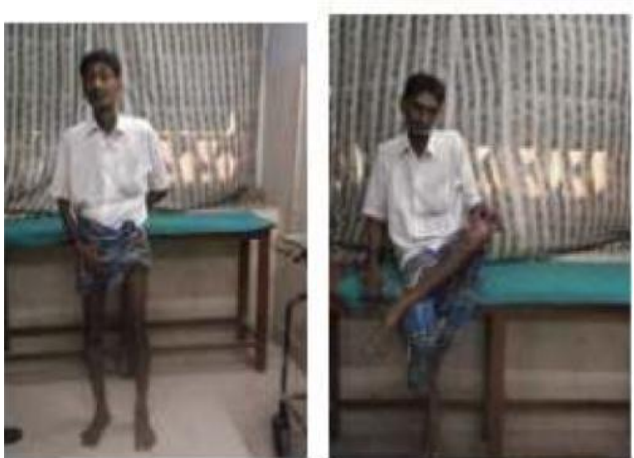

Fig. 2:

\section{Discussion}

Suman et al in $2017^{33}$ found that D.H.S had less surgical time less blood loss. Fisal and Nistane et al $(2016)^{21}$ found that P.F.N. had less intra operative blood loss. Singla et $\mathrm{al}^{31}$ found that P.F.N. had less amount of intra operative blood loss.

In our study surgical time and blood loss were greater in D.H.S group. Even though it was minimally invasive, the length of incision was longer in D.H.S group.

The main advantages of PFN are that there is only required shorter exposure required than the sliding screw and has lesser possibility of morbidity and operating time. PFN has immediate stability and good with unstable type of fractures (Babar et al., 2011). A study reported that with the 40 patients that were covered for Inter-trochanteric fracture, it derives that PFN has many advantages over these is sues.

In our study, the P.F.N group fractures healed fully earlier than D.H.S group for both stable and unstable fractures. The unstable fractures took more than $50 \%$ meantime of stable P.F.N group to unite fully. (Ramanarayananan ${ }^{20}$ )

Complications wise, there was not much of difference.

Nizamoddin Khateeb \& Babu, ${ }^{36}$ found in the treatment for inter trochanteric fractures the PFN has less operative blood loss, less complication, early return to daily activities and less sliding when compared to DHS treatment. 
Our study mirrors their finding.

\section{Conclusion}

The D.H.S had been the gold standard of intertrochanteric fractures. But P.F.N results also showed good results.

Considering all those analysed mentioned above we compared our results with the published results of D.H.S. Vs P.F.N. in trochanteric fractures. ${ }^{7,13,20,21,23,27,28,33}$ We conclude both D.H.S and P.F.N are good. But with shorter surgical time, less blood, quicker union time, the P.F.N is a better device to treat inter-trochanteric fractures, especially in unstable fractures.

\section{References}

1. Anand B. Jabshetty. Management of inter trochanteric fracture by DHS Fixation Indian. Journal of Science and Technology 2011;4(12).

2. Albareda J, Laderiga A, Palanca D et al Complications and technical problems with the gamma nail. Int Orthop1996; 20: 47-50.

3. Al-Yassari G, Langstaff RJ, Jones JW, Al-Lami M. The AO/ASIF proximal femoral nail (PFN) for the treatment of unstable trochanteric femoral fracture. Injury 2002;33 395-399.

4. Anne AK, Ekeland A, Odegaard B et al. Gamma nail versus compression screw for trochanteric femoral fracture. Act a OrthopScand1994;65:127-130

5. AngSen JO. Inter-trochanteric osteotomy for failed internal fixation of femoral neck fracture. Clin Orthop. 1997;341:175-182.

6. Babhulkar Sudhir S. Management of trochanteric Fractures Department of Orthopaedics, Indira Gandhi Medical College, Nagpur, Indian Journal of Orthopaedics October 2006 volume 40: Number 4: P. 210-218.

7. Bhakat U and Bandyopadhayay R. Comparative Study between Proximal Femoral Nailing and Dynamic Hip Screw in Inter-trochanteric Fracture of Femur. Open Journal of Orthopedics 2013;3(7):291-295.

8. Banan H, Al-Sabti A, Jimulia T, Hart AJ.The treatment of unstable, extracapsular hip fractures with the AO/ASIF proximal femoral nail (PFN) — our first 60 cases. Injury 2002;33:401-5.

9. Bartonicek J, Dousa P, Skala-Rosenbaum J, Kost'dl R. Soubornyreferat Trochanteric fractures. A current concepts review], Urazovachirurgie 2,001.

10. Blatter G, Janssen M. Treatment of subtrochanteric fractures of the femur: reduction on the traction table and fixation with dynamic condylar screw. Arch Orthop Trauma Surg 1994;113:138-41.

11. Baumgaertner MR, Curtin SL, Lindskog DM. Intramedullary versus extrameduilary fixation for treatment of Inter-trochanteric hip fractures. Clin Orthop Relat Res 1998;348:87-94.

12. Baumgaertner MR, Curtin SL, Lindskog DM, Keggi JM. The value of Tip-Apex distance, in predicting failure of fixation of peritrochantericfractures of the hip. J Bone Joint Surg (Am). 1995;77:1058-1064.

13. Baums HM, Eckert M, A comparative study of unstable per- and Inter-trochanteric femoral fractures treated with dynamic hip screw (MINIMAL INVASIVE DHS) and trochanteric butt- press plate vs. proximal femoral nail (PFN) ZentralblChir - Aug 2005.
14. Berman AT, Metzger PC, Bosacco SJ et al. Treatment of the subtrochanteric fractures with the compression hip nail: a review of 38 consecutive cases. Orthop Trans. 1979;3:255.

15. Bergman GD, Winkist RA, Mayo KA, Hanson SE. Subtrochanteric fractures of the femur: fixation using the Zickel nail. J Bone Joint Surg (Am). 1987;69A:10321040.

16. Baumgaertner MR, Curtin SL, Lindskog DM, Keggi JM. The value of Tip-Apex distance, in predicting failure of fixation of peritrochantericfractures of the hip. JBone Joint Surg (Am). 1995;77:1058-1064.

17. 17Cirotteau.y., Boyd H.B. and Griffin L.L. classification: A refinement proposal., European Journal of Orthopaedic Surgery \& Traumatology., January 2002, Volume 12, Issue 3, pp 152-157.

18. Evans, E. M. The treatment of trochanteric fractures of the femur. J. Bone Jt Surg. 1949;31-B:190-203.

19. Esser MP, Kassab JY, Jones DH. Trochanteric fractures of the femur. A randomised prospective trial comparing the Jewett nail-plate with the dynamic hip screw J Bone Joint Surg Br. 1986 Aug;68(4):557-60

20. Jonnes C, SM S, Najimudeen S. Type II Intertrochanteric Fractures: Proximal Femoral Nailing (PFN) Versus Dynamic Hip Screw (DHS). Archives of Bone and Joint Surgery2016;4(1):23-28.

21. Faisal M, Nistane P. Proximal Femoral Nailing vs. Dynamic Hip Screw in unstable Inter-trochanteric Fracture of Femur - A comparative analysis, International Journal of Biomedical and Advance Research 2016;7(10):489-492.

22. I. Kempf, A. Grosse, G. Taglang, E. Favreul Le clou gamma dans le traitement à foyer fermé des fractures trochantériennes. Résultats et indications à propos d'une série de 121 cas Revue de Chirurgie Orthopédique et Traumatologique, Volume 100, Issue 1, February 2014, Pages 70-79.

23. Klinger H M, BaumsHM, EckertM, and NeugebauerR. A comparative study of unstable per and Inter-trochanteric femoral fractures with DHS and PFN and T SP; Zentralblchir, 2005; 130.

24. Leung K S, So W S, Shen W Y, Hui P W. Gamma nails and dynamic hip screws for peritrochanteric fractures. $J$ Bone Joint Surg (Br) 1992;74:345-51.

25. Laros GS, Moore JF. Complications of Fixation in Intertrochanteric Fractures. Clin Orthop. 1974;101:110-9.

26. Moein CM, Verhofstad. MHJ, Bleys RLAW, Werken C van der (2005) Soft tissue injury related to the choice of entry point in ante grade femoral nailing; pyriform fossa or greater trochanter.

27. Pajarinen J, Lindahl J, Michelsson O, et al. Pertrochanteric femoral fractures treated with a dynamic hip screw or a proximal femoral nail: a randomised study comparing postoperative rehabilitation. J Bone Joint Surg Br. 2005;87:76-81.

28. Ramnarayan V, Vanchi PK, Kumar MM. Intra-medullary or Extra-medullary fixation for Inter-trochanteric fract ures - A comparison study. IOSR Journal of Dental and Medical Sciences (IOSR-JDMS) 2015;14(9), 15-21.

29. Sahu SK, Parichha K. Evaluation of results of "short proximal femoral nailing" in unstable trochanteric fractures. Journal of Indian Orthopaedic Rheumatology Association; 2016:2(1);15-27.

30. Sahlstrand T. The Richards compression and sliding hip screw system in the treatment of intertrochanteric fractures. Acta Orthop Scand. 1974;45(2):213-9. PubMed PMID: 4406976 
31. Singla G. A comparative study of 70 cases of intertrochanteric fracture femur treated with dynamic hip screw and proximal femoral nailing. Int J Res Orthop 2017;3:293-7.

32. Sonawane DV. Classifications of Inter-trochanteric fractures and their Clinical Importance. Trauma International 2015;1(1):7-11

33. Suman SK, Singh SKK and Manjhi LB. Proximal femoral nailing versus dynamic hip screw device for trochanteric fractures - A comparative study. International Journal of Orthopaedics Sciences 2017;3(2):738-740.

34. Synthes.vo.llnwd.net/o16/LLNWMB8/...PDF/DSEMTRM-0416-0657-1_LR.pd

35. http://synthes.vo.llnwd.net/o16/LLNWMB8/INT\%20Mo bile/Synthes\%20International/Product $\% 20$ Support $\% 20 \mathrm{M}$ aterial/legacy_Synthes_PDF/036.000.463.pdf

36. Khateeb MKN et al. Int J Res Orthop. 2017 May;3(3):602-606 International Journal of Research in Orthopaedics, May-June 2017,Vol 3, Issue 3 Page 603.

37. Edwards KP, et al. Modified Harris Hip score seen as accurate determinant of patient clinical outcome after THA., J Arthroplasty.

2016;doi:10.1016/j.arth.2015.10.016. 